Kalpa Publications in Engineering
Volume 1, 2017, Pages 19-25
ICRISET2017. International Conference on Re-
search and Innovations in Science, Engineering
\&Technology. Selected Papers in Engineering

\title{
Quantization \& Speech Coding for Wireless Communication
}

\author{
R.N.Rathod ${ }^{1}$, Dr. M.S.Holia ${ }^{2}$ \\ ${ }^{1}$ Assistant Professor, Power Electronics, Lukhdhirji Engineering College, Morbi, India, \\ ${ }^{2}$ Assistant Professor,EC Department, B.V.M. Engineering College, Anand, India \\ rathod_45@yahoo.com,msholia@bvmengineering.ac.in
}

\begin{abstract}
Quantization is basically the method of mapping a big set of input values to a smaller set, widely utilized in almost all the digital signal processing as it represents the signal in digital form. Aspect of data compression or coding is to shrink the number of bits necessary to deliver analog data, dependent on a distortion or fidelity conditions. In a wired communications very large bandwidth (range of frequency) are obtainable due to enormous bandwidth availability of optical fiber cables, while in a wireless communications range of frequency spectrum availability is a significant matters and service provider are continuously in penetrating of coder for obliging more consumers within a limited allocated spectrum. To achieve high speech quality at a lower bit rate coding algorithms plays a vital role to remove irrelevant information (redundancy) from speech signal. Good-looking progress have been made during last decades in code the speech signal with high quality at low bit rate for next generation wireless communication. This 1 paper provides the fundamentals of scalar $\&$ vector quantizers applied for analysis and synthesis of speech signal. This paper also includes the review regarding the various types of coders utilized in next generation wireless communication as well as current capability in research direction in speech coding techniques.
\end{abstract}

Keywords-Scalar quantization, Vector quantization, Codebook, Euclidean distance, Centroid, Mean Opinion Score.

\section{I.INTRODUCTION}

Major feature of wireless communication is to badge human being to correspond at anytime and anywhere by providing unlimited reach ability and accessibility [1].speech coding is the process of attaining condensed illustration of voice signals for proficient communication over band limited wireless channel and/or storage. It is also classified as the bit rate reduction of digital speech representation for transmission or storage[2].The speech coding in a simple words defined as characterize the speech signal with a least digits, Whereas upholding a enough level of quality of synthesized speech with equitable complications[3].Most of applications necessitate digitized

voice signal. Even though illustration of voice signal digitization fetches benefit of giving out it through hardware, it requires great data rate and that's why great requirement of bandwidth and storage.[4]. In Multimedia and some other speech related application where it is essential to 
accumulate digitized voice, speech coder plays a animated role to bridge the necessity of storage [5].Less memory, less bandwidth(range of frequency)requirements make it an attractive field for researcher and investigator. Cellular digital packet data (CDPD), different types of telephony, VOIP, are various modern applications of speech [6].

While we discuss coding techniques then Quantization play a vital role in unscrambling the additive noise from the signals. Sampling takes a snapshot of input signal and then sampled analog signal must be transformed to typical digital binary value from a voltage value that the machine can understand. Quantization maps a big set of input values to a smaller set. This paper is structured as follows: Section II designates types of Quantization. Section III represents structure of coding system. Section IV delivers current Capabilities in Speech Coding. Section V gives review on various types of coder utilized for next generation wireless communication and section VI concludes the reviews.

\section{TYPES OF QUANTIZATION}

Quantization forms the heart of any lossy compression scheme. Quantization encompasses representation of a set of fine resolution data by a coarse resolution approximation. Methods of Lossy compression can be approximately classified into two types: namely Scalar Quantization (SQ) and Vector Quantization (VQ).

Scalar quantization is used primarily for digital output form analog input, whereas vector quantization is used with sophisticated signal processing in which data is in digital form, SQ contains processing individually whereas VQ includes processing the input samples in sets (Groups) According to the theory of Rate Distortion provided by Claude E. Shannon when we process block of data as vectors rather than processing the data individually good use of the information of the signal search is achieved.

Figure-1 illustrates basic block diagram of a Vector Quantizer.The Vector quantization is powerfully associated to the reduction of the number of required bits necessary to convert continuous signals[11]. A VQ works as an approximator analogous to that of rounding off to nearest integer [7].Vector quantization (VQ) has become a valuable and essential technique in speech coding, where it is a part of industry standards and is in a wide commercial use [8]. Basic problems associated with vector quantizer are (i) it's large encoding complexity (ii) its kindliness to channel errors. The results obtained with vector quantization be contingents greatly on the codebook design strength and deign of encoder expresses the finest matching of codebook[9]. Basic structure of VQ is shown in Figure-2.as depicted in diagram. Vector quantization partitions the input space into $K$ non-overlapping regions in such a way that input space is completely covered. Code-vector is then allocated to each cluster. Vector quantization maps each input vector to code-vector, which is the demonstrative vector of the partition. usually the space is partitioned so that each vector is mapped to its nearest code-vector minimizing certain distortion function. The distortion is commonly the Euclidean distance between the two vectors:

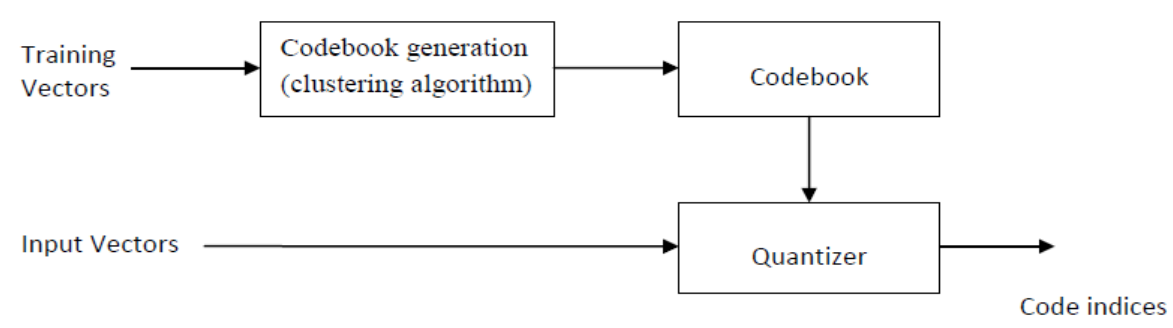


Figure-1: Basic block diagram of a Vector Quantizer [7]

$$
d(X, Y)=\sqrt{\sum_{i=1}^{M}\left(X_{i}-Y_{i}\right)^{2}}
$$

In above equation $X$ and $Y$ are two $M$-dimensional vectors. The code-vector is generally elected as the centroid of the vectors in the partition, that is

$$
C=\left(c_{1}, c_{2}, \ldots, c_{m}\right)=\left(\overline{X_{1}}, \overline{X_{2}}, \ldots, \overline{X_{m}}\right)
$$

where $X_{i}$ is the average value of the $i^{\text {th }}$ component of the vectors suitable to the partition diminishes the Euclidean distortion within the partition. The codebook of vector quantization consists of all the code-words[7]. The independent quantization of each single value or parameter is termed Scalar Quantization (SQ), whereas joint quantization of a group of parameters is termed Vector Quantization (VQ). Since 1980, Vector Quantization (VQ) becomes a popular technique for source coding of speech data.

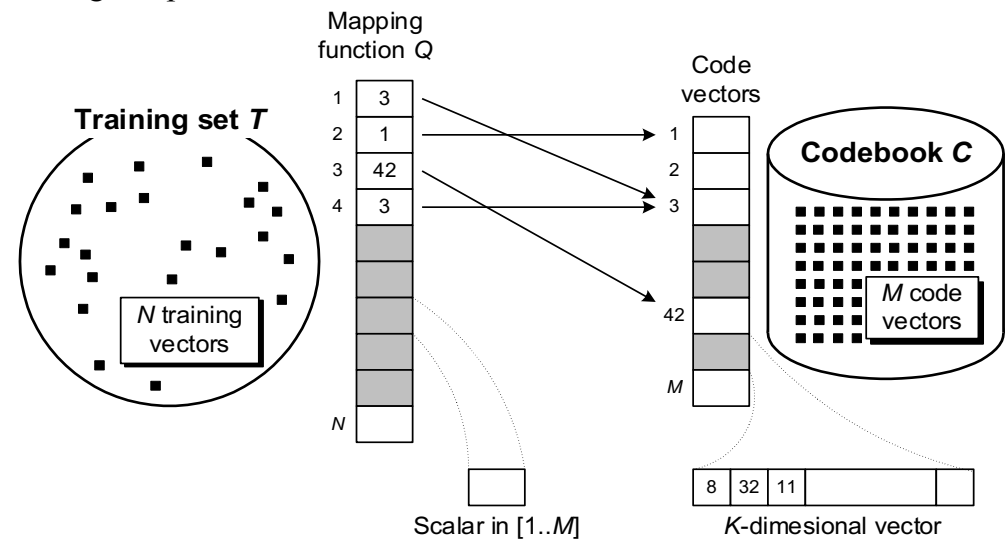

Figure-2: Basic structure of VQ [7]

\section{SPEECH CODING SYSTEM COMPONENTS}

Figure-3 illustrates basic components of speech coding system. In a Initial stage $\mathrm{s} \mathrm{p} \mathrm{e} \mathrm{e} \mathrm{ch}$ signal is acquired from the mike converts the acoustic pressure wave signal into typical electrical signals. Filter digitized the analog continuous time signal and pass to the sampler and analog to digital converter (ADC). Sampler converts the continuous time Analog speech signal to discrete time speech signal by taking samples of analog speech at nyquist rate.then Analog to digital converter discretize the sample values [17]. 


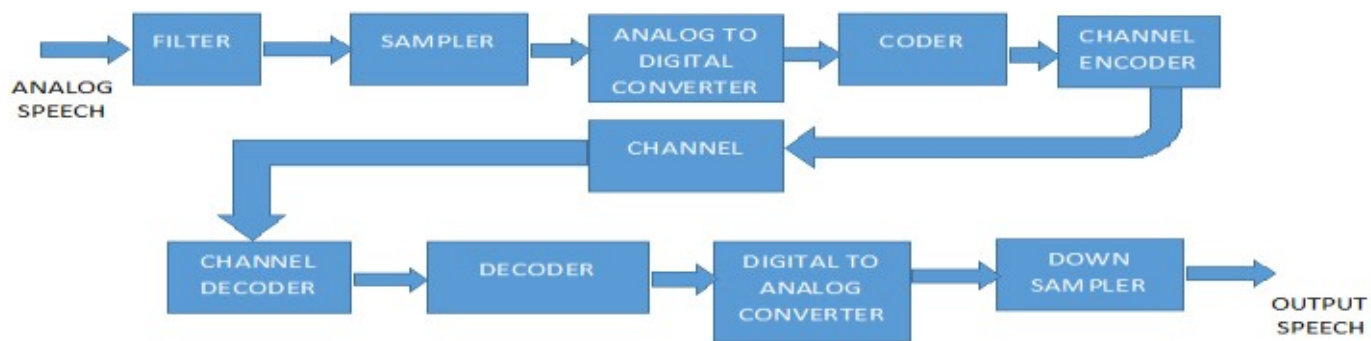

Figure-3: Speech Coding System [20]

The discretization of continuous speech signal using less than 20 bits per sample results in digital signal of less than $256 \mathrm{kbps}$ which is challenging to separate from original continuous speech signal. The data rate of original digital speech is actually the input data rate which speech coder endeavors to shrink at lower data rate. The encoded bit stream is given to the decoder which constructs an approximation to the original signal.

\section{CURRENT COMPETENCES IN SPEECH CODER}

Figure 4: demonstrates speech quality vs. MOS for different data rates. PCM works on 64kbps data rate is a simple utilized coding scheme of telephone speech signal. ADPCM (Adaptive differential Pulse code modulation) works at $32 \mathrm{kbps}$ used for increase the number of speech channels by a factor of two due to half the data rate. [4].for a data rate of less than $16 \mathrm{kbps}$ we can utilize regular-pulse excitation, multi-pulse excitation, code-excited linear prediction (CELP) coders, amongst that CELP coder is the best choice for broadcast of voice signal over telephone lines.

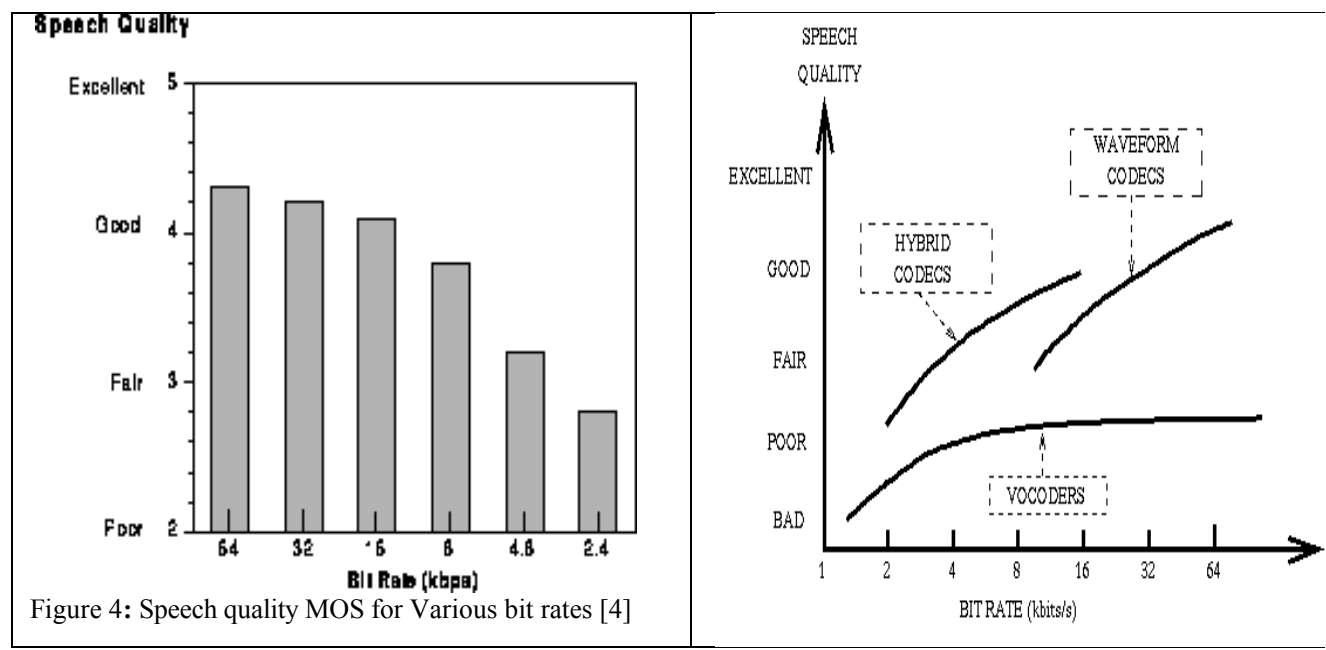

At bit rate lower than $8 \mathrm{kbps}$ A variant of CELP coder like LD-CELP, ACELP coder can be utilized. Before decade's we cannot produce high quality speech signal below data rate of $22 \mathrm{kbps}$. Now a day one can offer high quality speech at less than $8 \mathrm{kbps}$, making this a standard data rate for the next generation wireless communication. Utilizing good techniques for channel coding \& equalization one can transmit the less than $10 \mathrm{kbps}$ speech over radio(RF) channel in spite of multipath propagation and inter symbol interference[13].In Multimedia communications, digital simultaneous 
voice and data (DSVD), VOIP applications where storage requirement is a critical issue, speech coding become helpful[10]. ACELP based speech coder utilized in speech communication systems, accepted as a ideals owing to the twice advantage of data rate and speech quality[14].The CELP based AMR-WB speech coding algorithm has significant importance both for wireless as well as wire line services. and gives superior speech quality and voice spontaneity for next generation wireless communication [15]. CELP coder utilized in wireless communication based on latest ITU-T Recommendation scrambles voice signal in frames using Linear Prediction and analysis [20].

\section{REVIEW OF VARIOUS TYPES OF CODER}

Table 1 provides the reviews for various types of wireless standards, from which we can easily evaluate the difference between various standards.

\begin{tabular}{|c|c|c|c|c|}
\hline & & & & \multirow{14}{*}{$\begin{array}{l}\text { Synthesized speech of "s1ofwb" using LPC algo } \\
\text { igure } 1 \text { Original and synthesis speech of wave file } \\
\text { s1 ofwb" using LPC algorithm }\end{array}$} \\
\hline standard & & type used & & \\
\hline GSM & Cellular & RPE-LTP & $9.6,13$ & \\
\hline CD-900 & Cellular & SBC & 16 & \\
\hline $\begin{array}{l}\text { USDC (IS- } \\
136 \text { ) }\end{array}$ & Cellular & VSELP & 8 & \\
\hline IS-95 & Cellular & CELP & $\begin{array}{l}\text { 1.2,2.4,4.8, } \\
9.6,13.4,14.4\end{array}$ & \\
\hline IS-95 PCS & PCS & CELP & $13.4,14.4$ & \\
\hline PDC & Cellular & VSELP & $4.5,6.7,11$ & \\
\hline $\mathrm{CT} 2$ & Cordless & ADPCM & 32 & \\
\hline DECT & Cordless & ADPCM & 3 & \\
\hline PHS & Cordless & ADPCM & 32 & \\
\hline DCS-1800 & PCS & RPE-LTP & 13 & \\
\hline PACS & PCS & ADPCM & 32 & \\
\hline \multicolumn{4}{|c|}{$\begin{array}{l}\text { Table 1: Speech coders Used in Various First } \\
\text { and Second Generation Wireless System [4]. }\end{array}$} & \\
\hline
\end{tabular}




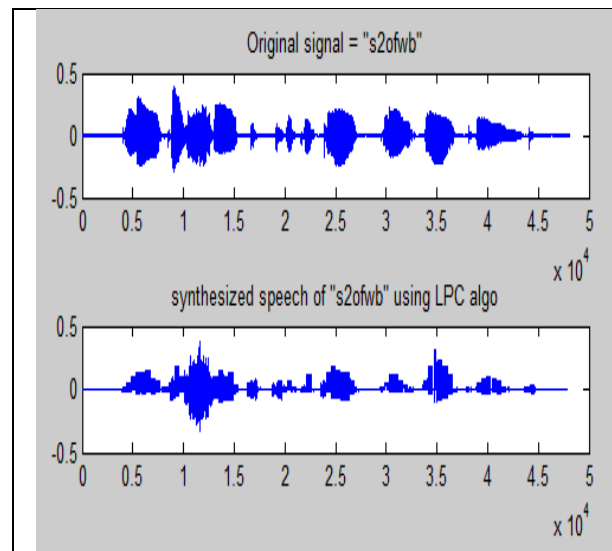

Figure 2 Original and synthesis speech of wave file "s2ofwb" using LPC algorithm

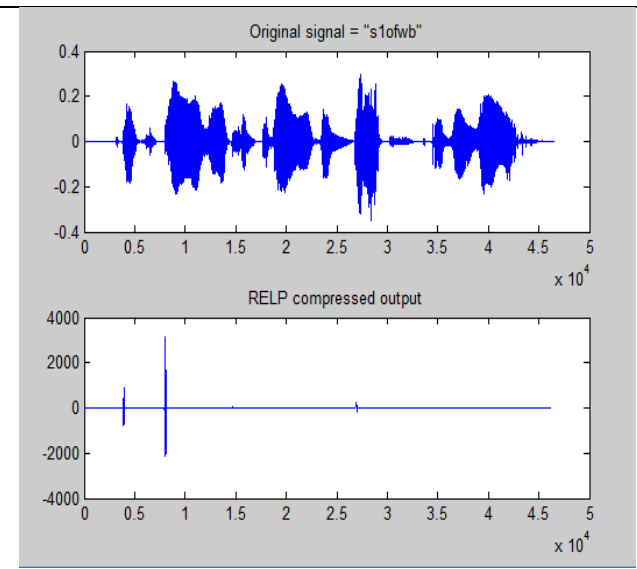

Figure 5 Original and synthesis speech of wave file "s1ofwb" using RELP algorithm

VI. Conclusion

From Simulation results one can say that the algorithms can be effectively applied to lowrate speech coding below 600bps. To remove the redundancy and transmit the speech with satisfactory quality, speech compression algorithms are positioned. Because of this reason the speech coding is and will be the most important research issue. Now a days due to advancement in signal processing\& VLSI technology it is possible to implement code and decoder on single chip. David Weenink \& Paul Boersma have proposed Praat software for speech, audio file analysis Source Forge has projected the Sound exchange software has agreed the client the ability to easily perform file conversions [21].

\section{Acknowledgment}

The Author would desire to thank Dr. M.S.Holia,Assistant Professor at B.V.M. Engineering College for his valuable comments and support.author would also like to thank Prof.M.V.Makwana, Associate Professor at L.E.College, Morbi for providing valuable suggestions.

\section{References}

[1]T.Rappaport,"Wireless Communications: Principles and Practice",Prentice-Hall, 1996. 
[2] B. S. Atal and M. R. Schroeder, "Predictive Coding of Speech Signals andSubjective Error Criteria" Ieee Trans. Speech, Signal Proc., Vol. 27, Pp.247 -254, 1979.

[3] A.S.Spanias,"Speech Coding: A Tutorial Review, Proc.Ieee, 82(1994),1541-1582.

[4]V.Garg, K.Smolik and J Wilkes, "Applications of CDMA inWireless/Personnel communications", Prentice-Hall, 1997. [7] M.H.Johnson and A.Alwan,"Speech Coding Fundamentals and Applications", Willey, December 2002.

[5] A. R. Sahab, M. Khoshroo, Islamic Azad University Lahijan ranchr.

[6] A.S.Spanias,"Speech Coding: A Tutorial Review, Proc.Ieee, 82(1994),1541-1582.

[7] Buzo, A., Gray, A.H., Gray, R.M., Markel, J.D., "Speech coding based upon vector quantization". IEEE Trans. Acoustic. SpeechSignal Process. pp:562-574, Year-1980.

[8] Linde Yoseph, Buzo Andrés, Gray Robert M.," An Algorithm for Vector Quantization", IEEE Transactions on Communications, Vol. COM-28, No. 1, pp. 84-95, January 1980.

[9] M.V.Makwana A.B.Nandurbarkar, S.M.Joshi” Image Compression using Tree Structured Vector Quantization with Compact Code - book ".IEEE Transaction on Comp \& Multimedia pp:102-107,2007.

[10] L.R.Rabiner and R.W.Schafer,"Digital Processing Of Speech Signals", Englewoo \&Caiffs, New Jersey, Prentice Hall, 1978.

[11]Sangeeta Ramakrishnan, Kenneth Rose , and Allen Gresho "Constrained-Storage Vector Quantizer with a Universal Codebook" IEEE Transactionson Image Pro., Vol.7, No. 6, pp. 785-793, June 1998.

[12]Pamela C.Cosman, Robert M.Gray and Martin Vetterli, "Vector Quantization of Image Subbands: a Survey", IEEE Transactions on Image Pro., Vol.5, No. 2, pp. 202-225, February 1996.

[13] ITU-Recommendation G.729, coding of speech at $8 \mathrm{~kb} / \mathrm{s}$ using conjugatestructure Algebraic code excited linear prediction (CS-ACELP).

[14] V.C.Togadiya, N.N.Shah, R.N.Rathod Comparative Review Between CELP And ACELP Encoder For CDMA, International Journal of Advanced Research in Electrical, Electronics and Instrumentation Engineering Vol. 2,

Issue 1, January 2013.

[15] N.Bhatt, Y. Kosta. “ overall performance Evaluation of Adaptive Multi Rate 6.90 speech codec based on CELP algoridhm using MATLAB”, International journal of speech technology springer, jan-2012.

[16]ITU-T Recommendation G.729, "Coding of speech at8kbps using conjugate-structure algebraic code excited linear prediction (CS-ACELP)", Dec 1995.

[17]Sanjeev Gupta,Science of Speech Coding,DRDO Science Spectrum, March 2009 pp. 120-127@ 2009, DESIDOC.

[18]Alan McCree ,LOW-BIT-RATE SPEECH CODING Springer Handbook on Speech Processing and Speech Communication

[19] Dipesh Bhagat, Ninad Bhatt and Yogeshwar Kosta - Adaptive Multi-Rate Wideband Speech Codec based on CELP algorithm: Architectural study, Implementation \& PerformanceAnalysis - IEEE International Conference on CommunicationSystems and Network Technologies, pages547 - 551,11-13 May-2012.

[20]K. Ubul, A. Hamdulla and A. Aysa, "A Digital Signal Processingteaching methodology using Praat," IEEE 4th International Conferenceon Computer Science and Education,pp. 1804-1809,2009.

[21] Lani Rachel MatheAnalysis of LD-CELP coder output with SoundExchange and PRAAT software, 2014 IEEE International Conference on Advanced Communication Control and Computing Technologies (ICACCCT).

[22]Ninad S. Bhatt, Technologies Implementation and Overall Performance Evaluation of CELP based GSM AMR NB coder over ABE,Fifth International Conference on Communication Systems and Network, 978-1-4799-1797-6/15 \$31.00 ( 2015 IEEE DOI 10.1109/CSNT.2015.46.

[23] N.S. Jayant and P.Noll. Digital coding of Wave forms, Prentice-Hall, Englewood Cliffs, New Jersey, 1984. 